\title{
Analysis of Li Ion Battery Anodes Using In-situ FIB-ToF-SIMS
}

Vincent Smentkowski ${ }^{1}$, Richard Hart ${ }^{1}$, Hongbo Cao ${ }^{1}$, Felix Kollmer ${ }^{2}$, Julia Zakel ${ }^{2}$ and Henrik Arlinghaus $^{2}$

${ }^{1}$ GE Research, Niskayuna, New York, United States, ${ }^{2}$ IONTOF GmbH, Münster, Nordrhein-Westfalen, Germany

Depth profiling (1D or 3D) is often used to determine the depth distribution of species in a material. Depth profiling works well when the surface of a sample is smooth and when the depth(s) are in the nm to micron thickness range. For thicker layers, one can mount the material in epoxy and generate a cross section which can be imaged. Unfortunately, cutting and polishing often damages (or at least smears) fragile materials such Li ion batteries. Over the two past decades, Focused Ion Beam (FIB) has proven to be a viable approach to expose sub surface layers 10 s of microns thick which is often analyzed by SEM or TEM in conjunction with EDS [1]. Unfortunately, most EDS detectors are not able to detect light species such as Li and C (especially at the degraded vacuum which most SEM's operate under), and definitely can not detect H. ToF-SIMS not only detects all elements (and their isotopes), it also allows for the analysis of molecular fragments which is often critical for understanding the material. FIB/ToF-SIMS has been used to analyze other material systems $[2,3]$.

In this poster, we provide preliminary in-situ FIB/ToF-SIMS results which were generated on an anode extracted from a Li ion battery. Figure 1 shows the configuration of the ToF-SIMS instrument (M6, manufactured by IONTOF) used for the analysis reported here. A $30 \mathrm{kV} \mathrm{Ga}^{+}$ion beam was used to generate FIB cuts which exposed sub surface areas $\sim 60 \mathrm{~mm}$ deep. The ToF-SIMS analysis were performed using a $30 \mathrm{kV} \mathrm{Bi}^{+}$ion beam. The left panel of Figure 2 shows a few negative ion ToF-SIMS images of the FIB prepared cross section; the negative ion images are normalized to the total negative ion image to minimize topography and increase chemical contrast.

ToF-SIMS data contain a wealth of information as a full mass spectrum is saved at every voxel. ToFSIMS data are ideal candidates for multivariate statistical analysis (MVSA) which boils the massive amount of information into a handful of factors $[4,5]$. The right panel of Figure 2 shows four of the factor images which were generated using the Multivariate Curve Resolution (MCR) MVSA algorithm [4, 5], each factor image has a corresponding mass spectrum (not shown). Factor 1 reveals smearing from the FIB procedure, Factor 2 is enriched in $\mathrm{F}$ and $\mathrm{NaF}$, Factor 3 is enriched in hydrocarbons $\mathrm{C} 9$ and smaller, and Factor 4 is enriched in $\mathrm{O}, \mathrm{Cl}$, and $\mathrm{CxHy}(\mathrm{x}=\leq 3)$.

In order to simultaneously obtain high lateral resolution images at high mass resolution, analysis are planned using the delayed extraction data collection mode [6]. 

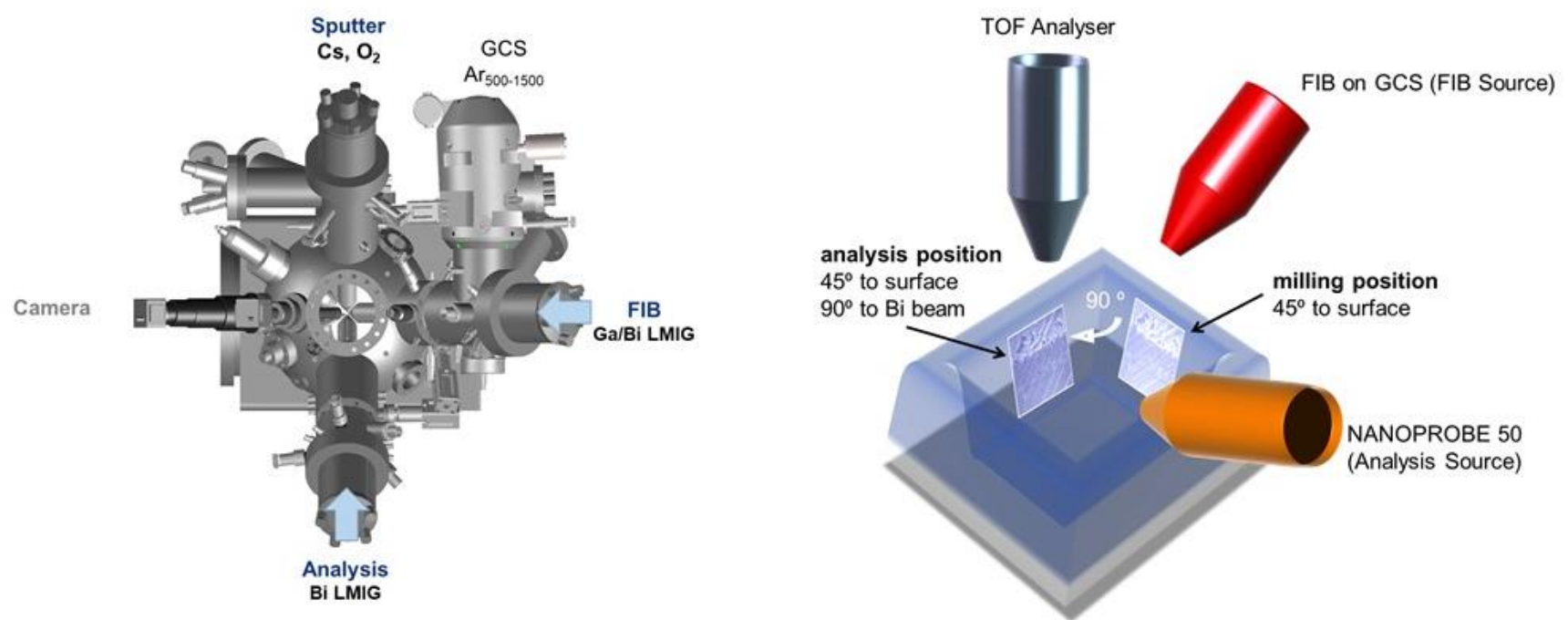

Figure 1. Top down view of the ToF-SIMS instrument (left panel) and sample orientation for both FIB preparation and ToF-SIMS analysis (right panel).

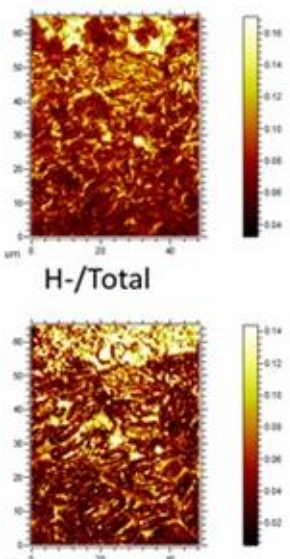

F-/Total

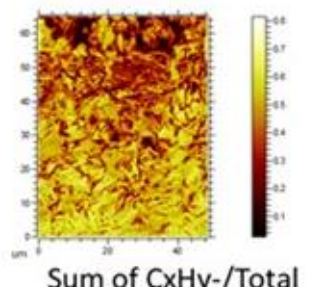

Sum of CxHy-/Total

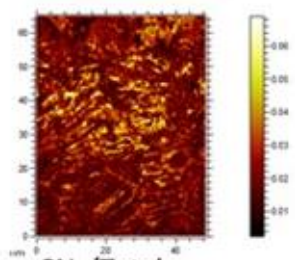

$\mathrm{CN}-/$ Total

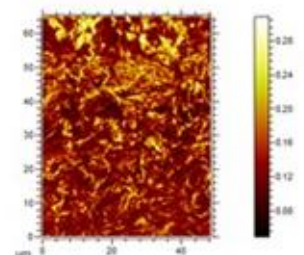

0-/Total

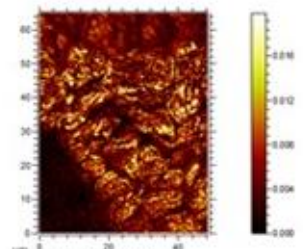

Cl-/Total

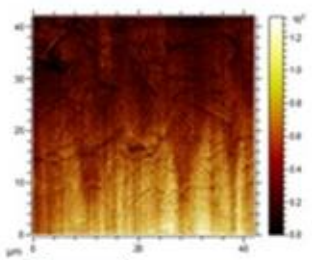

Factor 1

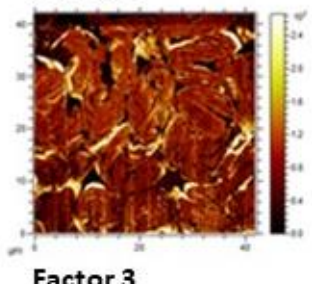

Factor 3

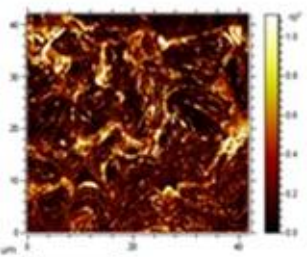

Factor 2

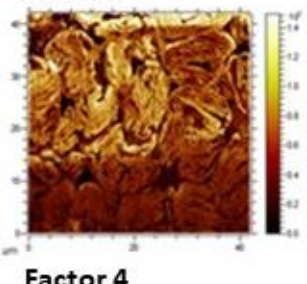

Figure 2. Negative ion ToF-SIMS images, which are normalized to the total ion image to reduce topography and increase chemical contrast (left panel) and four MVSA factor images (right panel), the corresponding spectra for each image is not shown.

\section{References}

[1] Lucille A. Giannuzzi, and Fred A. Stevie, Eds., "Introduction to Focused Ion Beams", Springer-Verlag US (2005) DOI: https://doi.org/10.1007/b101190.

[2] Felix Kollmer, D. Rading, R. Moellers, H.-G. Cramer, Wolfgang Paul, Ewald Niehuis, "Novel Cluster Ion Beams For Secondary Ion Generation, Sputtering And FIB/SIMS Application", Microscopy and Microanalysis 18(S2) (2012) 904-905; DOI: 10.1017/S143192761200637X.

[3] John S. Hammond, Gregory L. Fisher, Scott R. Bryan, Rait Kanarbik and Pritt Möller "FIB-TOF Tomography of Solid Oxide Fuel Cells", Microscopy and Microanalysis 19 (suppl 2) (2013) 672-673, DOI:10.1017/S1431927613005357.

[4] Vincent S. Smentkowski, Sara G. Ostrowski, Mike R. Keenan, "A Comparison of Multivariate Statistical Analysis Protocols for ToF-SIMS Spectral Images”, Surface and Interface Analysis, 41(2) (2009) 88. 
[5] Mike R. Keenan, Henrik Arlinghaus, Vincent S. Smentkowski "Using ToF-SIMS to study industrial surface phenomena" Surface Science 652 (2016) 39; DOI: 10.1016/j.susc.2016.02.017?

[6] Quentin P. Vanbellingen, Nicolas Elie, Michael J. Eller, Serge Della-Negra, David Touboul, Alain Brunelle, "Time-of-flight secondary ion mass spectrometry imaging of biological samples with delayed extraction for high mass and high spatial resolutions" Rapid Commun. Mass Spectrom. 29 (2015) 11871195, DOI: $10.1002 / \mathrm{rcm} .7210$ 